Proyecciones Journal of Mathematics

Vol. 34, No 4, pp. 379-390, December 2015.

Universidad Católica del Norte

Antofagasta - Chile

\title{
Computing the maximal signless Laplacian index among graphs of prescribed order and diameter
}

\author{
Nair Abreu* \\ Universidad Federal de Río de Janeiro, Brasil \\ Eber Lenes ${ }^{\dagger}$ \\ Universidad del Sinú, Colombia \\ and \\ Oscar Rojo $\ddagger$ \\ Universidad Católica del Norte, Chile. \\ Received : July 2015. Accepted : August 2015
}

\begin{abstract}
A bug Bug $g_{p, r_{1}, r_{2}}$ is a graph obtained from a complete graph $K_{p}$ by deleting an edge uv and attaching the paths $P_{r_{1}}$ and $P_{r_{2}}$ by one of their end vertices at $u$ and $v$, respectively. Let $Q(G)$ be the signless Laplacian matrix of a graph $G$ and $q_{1}(G)$ be the spectral radius of $Q(G)$. It is known that the bug $B_{0}=B u g_{n-d+2,\left\lfloor\frac{d}{2}\right\rfloor,\left\lceil\frac{d}{2}\right\rceil}$ maximizes $q_{1}(G)$ among all graphs $G$ of order $n$ and diameter $d$. For a bug $B$ of order $n$ and diameter $d, n-d$ is an eigenvalue of $Q(B)$ with multiplicity $n-d-1$. In this paper, we prove that remainder $d+$ 1 eigenvalues of $Q(B)$, among them $q_{1}(B)$, can be computed as the eigenvalues of a symmetric tridiagonal matrix of order $d+1$. Finally, we show that $q_{1}\left(B_{0}\right)$ can be computed as the largest eigenvalue of a symmetric tridiagonal matrix of order $\frac{d}{2}+1$ whenever $d$ is even.
\end{abstract}

Keyword : Signless Laplacian index, diameter, bug, H-join.

2000 AMS classification : 05C50, 05C35, 15A18.

${ }^{*}$ Thanks the support of Grant 305372/2009-2, CNPq, Brazil.

${ }^{\dagger}$ Thanks the support of Projects Mecesup UCN 0711, Mecesup UCN 1102 and Fondecyt Regular 1130135

${ }^{\ddagger}$ Thanks he support of Project Fondecyt Regular 1130135, Chile, and the hospitality of the Center For Mathematical Modeling, Universidad de Chile, Chile, in which this research was finished. 


\section{Introduction}

Let $G=(V(G), E(G))$ be a simple undirected graph of order $n$ with vertex set $V(G)$ and edge set $E(G)$. Let $D(G)$ be the diagonal matrix of order $n$ whose $(i, i)$ - entry is the degree of the $i$ - th vertex of $G$ and let $A(G)$ be the adjacency matrix of $G$. The matrices $L(G)=D(G)-A(G)$ and $Q(G)=D(G)+A(G)$ are the Laplacian matrix and signless Laplacian matrix of $G$, respectively. These matrices are both positive semidefinite matrices and they have the same characteristic polynomial if and only if $G$ is a bipartite graph. The eigenvalues of $A(G), L(G)$ and $Q(G)$ are called the eigenvalues, Laplacian eigenvalues and signless Laplacian eigenvalues of $G$, respectively. In particular, the spectral radius of $Q(G)$ is called the signless Laplacian index of $G$ and it is usually denoted by $q_{1}(G)$. From the Perron - Frobenius Theory for nonnegative matrices, it follows that if $G$ is a connected graph then $q_{1}(G)$ is a simple eigenvalue of $Q(G)$.

Let $K_{n}$ and $P_{n}$ be a complete graph and a path on $n$ vertices, respectively. A bug $B u g_{p, r_{1}, r_{2}}$ is a graph obtained from $K_{p}$ by deleting an edge $u v$ and attaching the paths $P_{r_{1}}$ and $P_{r_{2}}$ by one of their end vertices at $u$ and $v$, respectively. Observe that $B u g_{p, r_{1}, r_{2}}$ is a graph of order $p+r_{1}+r_{2}-2$ and diameter $r_{1}+r_{2}$.

Example 1. For instance $B u g_{6,3,4}$ is the graph

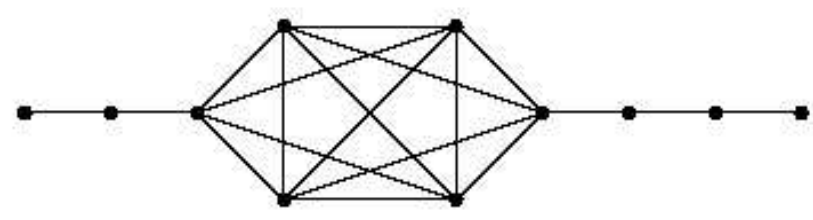

of 11 vertices and diameter 7 .

Let $\mathcal{G}_{n}$ be the class of all connected graphs on $n$ vertices and let $\mathcal{G}_{n, d}$ be the subclass of graphs in $\mathcal{G}_{n}$ with diameter $d$. Since $\mathcal{G}_{n, 1}=\left\{K_{n}\right\}$ and $\mathcal{G}_{n, n-1}=\left\{P_{n}\right\}$, throughout this paper, we assume $2 \leq d \leq n-2$. Let

$$
\mathcal{B}_{n, d}=\left\{\text { Bug }_{n-d+2, i, d-i}: 1 \leq i \leq d-1\right\} .
$$

Clearly $\mathcal{B}_{n, d}$ is a subclass of $\mathcal{G}_{n, d}$.

Some results such that $q_{1}(G)$ is maximal among graphs with fixed invariants are known. For instance, in [4] the graph having the largest $q_{1}(G)$ 
among the graphs with fixed numbers of vertices and edges is found, in [6] the graphs with the largest $q_{1}(G)$ and the largest adjacency index among all graphs with a fixed vertex connectivity or a fixed edge connectivity are characterized and in [7] the author characterizes the graphs having the largest $q_{1}(G)$ among all the graphs on $n$ vertices and a given matching number. of trees in $\mathcal{T}_{n, d}$ are characterized.

For a bug $B$ of order $n$ and diameter $d, n-d$ is an eigenvalue of $Q(B)$ with multiplicity $n-d-1$. In this paper, we prove that remainder $d+1$ eigenvalues of $Q(B)$ can be computed as the eigenvalues of a symmetric tridiagonal matrix of order $d+1$.

A conjecture proposed by Hansen and Lucas [5] states that, for a given $n \geq 9$, the bug $B_{\left\lfloor\frac{n}{2}\right\rfloor+1,\left\lfloor\frac{D}{2}\right\rfloor,\left\lceil\frac{D}{2}\right\rceil}$, where $D=\left\lceil\frac{n+1}{2}\right\rceil$, is the unique connected graph of order $n$ that maximizes the product $q_{1}(G) \operatorname{diam}(G)$ over all connected graphs $G$ of order $n$. This conjecture was studied by H. Liu and M. $\mathrm{Lu}[3]$. They proved that the bug $B_{0}=B u g_{n-d+2,\left\lfloor\frac{d}{2}\right\rfloor,\left\lceil\frac{d}{2}\right\rceil}$ maximizes $q_{1}(G)$ among all graphs $G$ of order $n$ and diameter $d$ and that, for a given $n$, the bug $B_{\left\lfloor\frac{n}{2}\right\rfloor+2,\left\lfloor\frac{D}{2}\right\rfloor,\left\lceil\frac{D}{2}\right\rceil}$, where $D=\left\lceil\frac{n+1}{2}\right\rceil$, is the unique connected graph of order $n$ that maximizes the product $q_{1}(G) \operatorname{diam}(G)$ over all connected graphs of order $n$.

Moreover, in this paper, we prove that $q_{1}\left(B_{0}\right)$ can be computed as the largest eigenvalue of a symmetric tridiagonal matrix of order $\frac{d}{2}+1$ whenever $d$ is even.

We recall the notion of the join operation of graphs. Given two vertex disjoint graphs $G_{1}$ and $G_{2}$, the join of $G_{1}$ and $G_{2}$ is the graph $G=G_{1} \vee$ $G_{2}$ such that $\mathrm{V}(G)=V\left(G_{1}\right) \cup V\left(G_{2}\right)$ and $\mathrm{E}(G)=E\left(G_{1}\right) \cup E\left(G_{2}\right) \cup$ $\left\{x y: x \in V\left(G_{1}\right), y \in V\left(G_{2}\right)\right\}$.

The join operation of two vertex disjoint graphs can be generalize as follows $[1,2]$. Let $H$ be a graph of order $k$. Let $V(H)=\{1, \ldots, k\}$ be the vertex set of $H$. Let $\left\{G_{1}, G_{2}, \ldots, G_{k}\right\}$ be a set of pairwise vertex disjoint graphs. For $1 \leq j \leq k$, the vertex $j \in V(H)$ is assigned to the graph $G_{j}$. Let $G$ be the graph obtained from the graphs $G_{1}, G_{2}, \ldots, G_{k}$ and the edges connecting each vertex of $G_{i}$ with all the vertices of $G_{j}$ if and only if $i j \in E(H)$. That is, $G$ is the graph with vertex set $\mathrm{V}(\mathrm{G})=\bigcup_{i=1}^{k} V\left(G_{i}\right)$ and edge set $\mathrm{E}(\mathrm{G})=\left(\bigcup_{i=1}^{k} E\left(G_{i}\right)\right) \cup\left(\bigcup_{i j \in E(H)}\left\{u v: u \in V\left(G_{i}\right), v \in V\left(G_{j}\right)\right\}\right)$. This graph is called the $H-$ join of the graphs $G_{1}, \ldots, G_{k}$ and it is denoted by $\mathrm{G}=\bigvee_{H}\left\{G_{j}: 1 \leq j \leq k\right\}$.

We see that if $n_{i}$ is the order of $G_{i}, i=1,2, \ldots, k$, then $H-j$ oin of $G_{1}, \ldots, G_{k}$ is a graph of order $n_{1}+n_{2}+\ldots+n_{k}$. 
Important examples of this graph operation are the bugs $B u g_{n-d+2, i, d-i}$. In fact, the bug $B u g_{n-d+2, i, d-i}$ is the $P_{d+1}-j$ oin of the regular graphs $G_{1}=\ldots=G_{i}=K_{1}, G_{i+1}=K_{n-d}, G_{i+2}=\ldots=G_{d+1}=K_{1}$.

Since $B u g_{n-d+2, i, d-i}$ and $B u g_{n-d+2, d-i, i}$, are isomorphic graphs, we may take $1 \leq i \leq\left\lfloor\frac{d}{2}\right\rfloor$.

Example 2. Below are the non-isomorphic bugs of order 11 and diameter 7.

Bug $_{6,1,6}$ is the $P_{8}-j$ oin of $G_{1}=K_{1}, G_{2}=K_{4}$ and $G_{i}=K_{1}$ for $i=3, \ldots, 8$ :

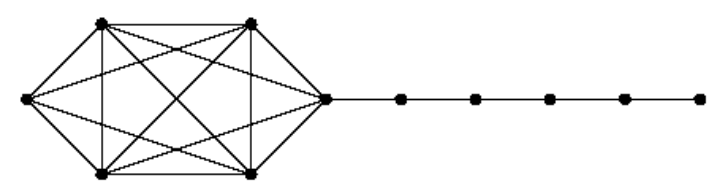

Bug $g_{6,2,5}$ is the $P_{8}-j$ oin of $G_{1}=G_{2}=K_{1}, G_{3}=K_{4}$ and $G_{i}=K_{1}$ for $i=4, \ldots, 8$ :

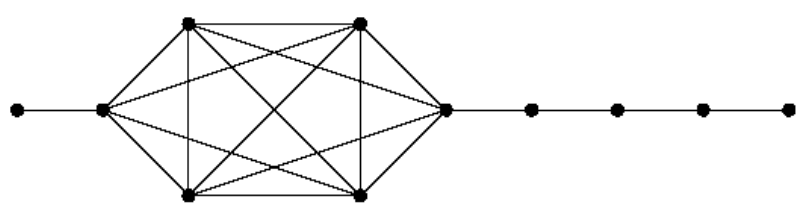

Bug $g_{6,3,4}$ is the $P_{8}-j$ oin of $G_{1}=G_{2}=G_{3}=K_{1}, G_{4}=K_{4}$ and $G_{i}=K_{1}$ for $i=5, \ldots, 8$ :

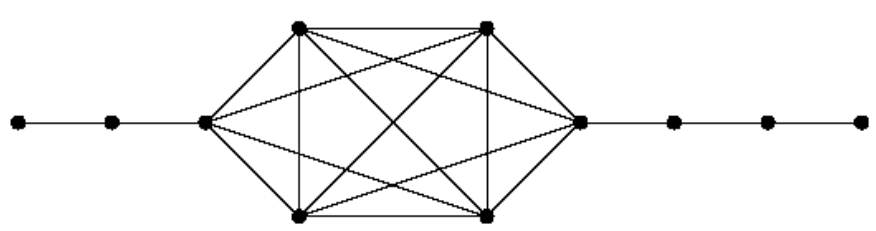




\section{The signless Laplacian eigenvalues of bugs}

In [1], Theorem 5, the spectrum of the adjacency matrix of the $H$ - join of regular graphs is obtained. The version of this result for the signless Laplacian matrix is given below and its proof is similar.

Theorem 1. Let $H$ be a graph with $k$ vertices. Let $G=\bigvee_{H}\left\{G_{j}: 1 \leq j \leq k\right\}$. For $j=1, \ldots, k$, let $G_{j}$ be a $r_{j}$-regular graph of order $n_{j}$. Then

$$
\sigma(Q(G))=\cup_{G_{j} \neq K_{1}}\left\{s_{j}+\lambda: \lambda \in \sigma\left(Q\left(G_{j}\right)\right) \backslash\left\{2 r_{j}\right\}\right\} \cup \sigma(M(G))
$$

where $M(G)$ is a matrix of order $k \times k$ given by

$$
M(G)=\left[\begin{array}{cccc}
s_{1}+2 r_{1} & \delta_{12} \sqrt{n_{1} n_{2}} & \ldots & \delta_{1 k} \sqrt{n_{1} n_{k}} \\
\delta_{12} \sqrt{n_{1} n_{2}} & s_{2}+2 r_{2} & \ddots & \vdots \\
\vdots & \ddots & \ddots & \delta_{(k-1) k} \sqrt{n_{k-1} n_{k}} \\
\delta_{1 k} \sqrt{n_{1} n_{k}} & \ldots & \delta_{(k-1) k} \sqrt{n_{k-1} n_{k}} & s_{k}+2 r_{k}
\end{array}\right]
$$

with

$$
\delta_{i j}=\left\{\begin{array}{c}
1 \text { if } i j \in E(H) \\
0 \text { otherwise }
\end{array}\right.
$$

and, for $j=1,2, \ldots, k$,

$$
s_{j}=\sum_{j l \in E(H)} n_{l}
$$

For brevity, let $B(i)=B u g_{n-d+2, i, d-i}$. Remember that we may take $1 \leq i \leq\left\lfloor\frac{d}{2}\right\rfloor$.

We already observed $B(i)=B u g_{n-d+2, i, d-i}$ is the $P_{d+1}-j$ oin of the regular graphs $G_{1}=\ldots=G_{i}=K_{1}, G_{i+1}=K_{n-d}, G_{i+2}=\ldots=G_{d+1}=$ $K_{1}$. Hence Theorem 1 can be applied to determine its signless Laplacian eigenvalues. For all the bugs $B(i)$, the graph $H$ in Theorem 1 is the path $P_{d+1}$. Hence the matrix $M(B(i))$ in $(2.2)$ becomes a symmetric tridiagonal 
matrix of order $(d+1) \times(d+1)$ :

$$
M(B(i))=\left[\begin{array}{cccccc}
s_{1}+2 r_{1} & \sqrt{n_{1} n_{2}} & & & & \\
\sqrt{n_{1} n_{2}} & s_{2}+2 r_{2} & \sqrt{n_{2} n_{3}} & & & \\
& \sqrt{n_{2} n_{3}} & s_{3}+2 r_{3} & \ddots & & \\
& & \ddots & \ddots & \ddots & \\
& & & \ddots & s_{d}+2 r_{d} & \sqrt{n_{d} n_{d+1}} \\
& & & & \sqrt{n_{d} n_{d+1}} & s_{d+1}+2 r_{d+1}
\end{array}\right] .
$$

For convenience, we define the matrices

Definition 1. Let

$$
\begin{gathered}
R(n-d)=\left[\begin{array}{ccc}
n-d+1 & \sqrt{n-d} & 0 \\
\sqrt{n-d} & 2(n-d) & \sqrt{n-d} \\
0 & \sqrt{n-d} & n-d+1
\end{array}\right] \\
T_{1}=[1]
\end{gathered}
$$

and, for $s \geq 2$, let

$$
T_{s}=\left[\begin{array}{ccccc}
1 & 1 & & & \\
1 & 2 & 1 & & \\
& 1 & \ddots & \ddots & \\
& & \ddots & 2 & 1 \\
& & & 1 & 2
\end{array}\right]
$$

of order $s \times s$.

Moreover, we introduce the following matrices: $I$ is the identity matrix, 0 is the zero matrix, $J$ is the exchange matrix (the matrix with ones in the secondary diagonal and zeros elsewhere) and $F$ is the matrix whose entries are zeros except for the entry in the last row and first column which is equal to 1 . The orders of these matrices will be clear from the context in which they are used.

Theorem 2. The eigenvalues of $B(i)=B u g_{n-d+2, i, d-i}$ are $n-d$ with multiplicity $n-d-1$ and the eigenvalues of the $(d+1) \times(d+1)$ symmetric tridiagonal matrix

$$
M(B(i))=\left[\begin{array}{cc}
X_{i} & F \\
F^{T} & J T_{d-i-1} J
\end{array}\right]
$$


where

$$
X_{1}=\left[\begin{array}{ccc}
n-d & \sqrt{n-d} & 0 \\
\sqrt{n-d} & 2(n-d) & \sqrt{n-d} \\
0 & \sqrt{n-d} & n-d+1
\end{array}\right]
$$

whenever $i=1$,

$$
X_{i}=\left[\begin{array}{cc}
T_{i-1} & F \\
F^{T} & R(n-d)
\end{array}\right]
$$

whenever $2 \leq i \leq\left\lfloor\frac{d}{2}\right\rfloor$ and $F$ is the matrix defined above.

Proof. We know that $B(i)=B u g_{n-d+2, i, d-i}$ is the $P_{d+1}-j$ oin of the regular graphs $G_{1}=\ldots=G_{i}=K_{1}, G_{i+1}=K_{n-d}, G_{i+2}=\ldots=G_{d+1}=$ $K_{1}$. Thus $G_{j}=K_{1}$ for all $j$ except for $j=i+1$. For $j=i+1$, we have $G_{i+1}=K_{n-d}$ which is a $(n-d-1)$ - regular graph. From (2.3), $s_{i+1}=n_{i}+n_{i+2}=1+1=2$. Then, from (2.1), we have

$$
\sigma(Q(B(i)))=\left\{2+\lambda: \lambda \in Q\left(K_{n-d}\right) \backslash\{2(n-d-1)\}\right\} \cup \sigma(M(B(i)) .
$$

At this point, we recall that the signless Laplacian eigenvalues of $K_{n-d}$ are $2(n-d-1)$ and $n-d-2$ with multiplicity $n-d-1$. Using this fact in $(2.9)$, we obtain $\sigma(Q(B(i)))=\left\{(n-d)^{[n-d-1]}\right\} \cup \sigma(M(B(i))$ where $(n-d)^{[n-d-1]}$ means that $n-d$ is an eigenvalue of multiplicity $n-d-1$.

We now search for the entries of $M(B(i))$ in (2.4). We begin with $M(B(1))$. The bug $B(1)$ is the $P_{d+1}-$ join of $G_{1}=K_{1}, G_{2}=K_{n-d}$ and $G_{3}=G_{4}=\ldots=G_{d+1}=K_{1}$. For this bug

$$
\begin{array}{cc}
n_{1}=1 & r_{1}=0 \\
n_{2}=n-d & r_{2}=n-d-1 \\
n_{3}=1 & r_{3}=0 \\
\vdots & \vdots \\
n_{d}=1 & r_{d}=0 \\
n_{d+1}=1 & r_{d+1}=0
\end{array}
$$

Then $s_{1}=n_{2}=n-d, s_{2}=n_{1}+n_{3}=2, s_{3}=n_{2}+n_{4}=n-d+1, s_{4}=$ $n_{3}+n_{5}=2, \ldots, s_{d}=n_{d-1}+n_{d+1}=2, s_{d+1}=n_{d}=1$. Replacing these values in (2.4), we obtain 


$$
\mathrm{M}(\mathrm{B}(1))=\left[\begin{array}{cc}
X_{1} & F \\
F^{T} & J T_{d-2} J
\end{array}\right] \text { with } \mathrm{X}_{1}=\left[\begin{array}{ccc}
n-d & \sqrt{n-d} & 0 \\
\sqrt{n-d} & 2(n-d) & \sqrt{n-d} \\
0 & \sqrt{n-d} & n-d+1
\end{array}\right]
$$

and $T_{d-2}$ as in (2.5). The theorem has been proved for $B(1)$. Let $2 \leq i \leq$ $\left\lfloor\frac{d}{2}\right\rfloor$. The bug $B(2)$ is the $P_{d+1}-$ join of the regular graphs $G_{1}=G_{2}=$ $K_{1}, G_{3}=K_{n-d}, G_{4}=\ldots=G_{d+1}=K_{1}$. For $B(2)$, we have

$$
\begin{array}{cc}
n_{1}=1 & r_{1}=0 \\
n_{2}=1 & r_{2}=0 \\
n_{3}=n-d & r_{3}=n-d-1 \\
n_{4}=1 & r_{4}=0 \\
\vdots & \vdots \\
n_{d}=1 & r_{d}=0 \\
n_{d+1}=1 & r_{d+1}=0
\end{array}
$$

Then $s_{1}=n_{2}=1, s_{2}=n_{1}+n_{3}=n-d+1, s_{3}=n_{2}+n_{4}=2, s_{4}=n_{3}+$ $n_{5}=n-d+1, s_{5}=n_{4}+n_{6}=2, \ldots, s_{d}=n_{d-1}+n_{d+1}=2, s_{d+1}=n_{d}=1$.

Replacing these values in (2.4), we get

$$
\mathrm{M}(\mathrm{B}(2))=\left[\begin{array}{cc}
X_{2} & F \\
F^{T} & J T_{d-3} J
\end{array}\right]
$$

where

$$
\mathrm{X}_{2}=\left[\begin{array}{cccc}
1 & 1 & 0 & 0 \\
1 & n-d+1 & \sqrt{n-d} & 0 \\
0 & \sqrt{n-d} & 2(n-d) & \sqrt{n-d} \\
0 & 0 & \sqrt{n-d} & n-d+1
\end{array}\right]
$$

and $T_{d-3}$ as in (2.5). The bug $B(3)$ is the $P_{d+1}-$ join of $G_{1}=G_{2}=G_{3}=$ $K_{1}, G_{4}=K_{n-d}, G_{5}=\ldots=G_{d+1}=K_{1}$. Similarly

$$
\mathrm{M}(\mathrm{B}(3))=\left[\begin{array}{cc}
X_{3} & F \\
F^{T} & J T_{d-4} J
\end{array}\right]
$$

where

$$
\mathrm{X}_{3}=\left[\begin{array}{ccccc}
1 & 1 & 0 & 0 & 0 \\
1 & 2 & 1 & 0 & 0 \\
0 & 1 & n-d+1 & \sqrt{n-d} & 0 \\
0 & 0 & \sqrt{n-d} & 2(n-d) & \sqrt{n-d} \\
0 & 0 & 0 & \sqrt{n-d} & n-d+1
\end{array}\right]
$$

and $T_{d-4}$ as in (2.5). We continue in this fashion obtaining that the result also holds for $i=4, \ldots,\left\lfloor\frac{d}{2}\right\rfloor$. 


\section{Computing the largest signless Laplacian index of graphs of prescribed order and diameter}

We already mentioned that H. Liu and M. Lu, Theorem 3.2 in [3] characterized the largest signless Laplacian index among the graphs in $\mathcal{G}_{n, d}$.

Theorem 3. Among all the graphs $G$ on $n$ vertices and diameter $d, 2 \leq d \leq$ $n-2$, the largest $q_{1}(G)$ is attained by the bug $B\left(\left\lfloor\frac{d}{2}\right\rfloor\right)=B u g_{n-d+2,\left\lfloor\frac{d}{2}\right\rfloor,\left\lceil\frac{d}{2}\right\rceil}$.

Theorem 3 tell us that the largest signless Laplacian index among the graph in $\mathcal{G}_{n, d}$ is $q_{1}\left(B\left(\left\lfloor\frac{d}{2}\right\rfloor\right)\right.$. From Theorem $2, q_{1}\left(B\left(\left\lfloor\frac{d}{2}\right\rfloor\right)\right.$ can be computed as the largest eigenvalues of the symmetric tridiagonal matrix $M\left(B\left(\left\lfloor\frac{d}{2}\right\rfloor\right)\right)$ of order $d+1$. More precisely

Theorem 4. Let $G \in \mathcal{G}_{n, d}$.

(a) If $d=3$ then the largest $q_{1}(G)$ can be computed as the largest eigenvalue of the symmetric tridiagonal matrix $M(B(1))$ of order 4 with diagonal entries

$$
n-3,2(n-3), n-2,1
$$

and codiagonal entries

$$
\sqrt{n-3}, \sqrt{n-3}, 1
$$

(b) If $d \geq 4$ then the largest $q_{1}(G)$ can be computed as the largest eigenvalue of the symmetric tridiagonal matrix $M\left(B\left(\left\lfloor\frac{d}{2}\right\rfloor\right)\right)$ of order $d+1$ with diagonal entries

$$
1, \overbrace{1,2, \ldots, 2}^{\left\lfloor\frac{d}{2}\right\rfloor-2}, n-d+1,2(n-d), n-d+1, \overbrace{2, \ldots, 2}^{\left\lceil\frac{d}{2}\right\rceil-2}, 1
$$

codiagonal entries

$$
\overbrace{1, \ldots, 1}^{\left\lfloor\frac{d}{2}\right\rfloor-1}, \sqrt{n-d}, \sqrt{n-d}, \overbrace{1, \ldots, 1}^{\left\lceil\frac{d}{2}\right\rceil-1} .
$$

We now prove that $q_{1}\left(B\left(\left\lfloor\frac{d}{2}\right\rfloor\right)\right.$ can be computed as the largest eigenvalue of a symmetric tridiagonal matrix of order $\frac{d}{2}+1$ whenever $d$ is an even integer. 
Theorem 5. If $d \geq 4$ is an even integer then among the graphs $G$ on $n$ vertices and diameter $d$, the largest $q_{1}(G)$ can be computed as the largest eigenvalue of a symmetric tridiagonal matrix of order $\frac{d}{2}+1$ with diagonal entries

$$
1,2, \ldots, 2, n-d+1,2(n-d)
$$

and codiagonal entries

$$
1, \ldots, 1, \sqrt{2(n-d)}
$$

Proof. Let $d$ be an even integer and $\alpha=n-d$. From Theorem 4, $q_{1}\left(M\left(B\left(\frac{d}{2}\right)\right)\right.$ can be computed as the largest eigenvalue of

$$
M\left(B\left(\frac{d}{2}\right)\right)=\left[\begin{array}{ccc}
U & \mathbf{b} & 0 \\
\mathbf{b}^{T} & 2 \alpha & \mathbf{b}^{T} J \\
0 & J \mathbf{b} & J U J
\end{array}\right]
$$

of order $d+1$ where

$$
U=\left[\begin{array}{ccccc}
1 & 1 & & & \\
1 & 2 & \ddots & & \\
& \ddots & \ddots & 1 & \\
& & 1 & 2 & 1 \\
& & & 1 & \alpha+1
\end{array}\right]
$$

of order $\frac{d}{2}, \mathbf{b}^{T}=\left[\begin{array}{lllll}0 & \cdots & \cdots & 0 & \sqrt{\alpha}\end{array}\right], J$ is the reverse matrix and 0 is the zero matrix, all of them of the appropriate sizes. Consider the orthogonal matrix

$$
Q=\frac{1}{\sqrt{2}}\left[\begin{array}{ccc}
I & \mathbf{0} & J \\
\mathbf{0}^{T} & \sqrt{2} & \mathbf{0}^{T} \\
-J & \mathbf{0} & I
\end{array}\right]
$$

An easy calculation shows that

$$
Q M\left(B\left(\frac{d}{2}\right)\right) Q^{T}=\left[\begin{array}{ccc}
U & \sqrt{2} \mathbf{b} & 0 \\
\sqrt{2} \mathbf{b}^{T} & 2 \alpha & \mathbf{0}^{T} \\
0 & \mathbf{0} & J U J
\end{array}\right] .
$$


Then the eigenvalues of $M\left(B\left(\frac{d}{2}\right)\right)$ are the eigenvalues of $\left[\begin{array}{cc}U & \sqrt{2} \mathbf{b} \\ \sqrt{2} \mathbf{b}^{T} & 2 \alpha\end{array}\right]$ and the eigenvalues of $U$. Since the eigenvalues of $U$ strictly interlace the eigenvalues of $\left[\begin{array}{cc}U & \sqrt{2} \mathbf{b} \\ \sqrt{2} \mathbf{b}^{T} & 2 \alpha\end{array}\right]$, the proof is complete.

\section{References}

[1] D. M. Cardoso, M. A. A. de Freitas, E. Martins., M. Robbiano, Spectra of graphs obtained by a generalization of the join graph operation, Discrete Mathematics 313, pp. 733-741, (2013).

[2] D. M. Cardoso, E. Martins., M. Robbiano, O. Rojo, Eigenvalues of a H-generalized operation constrained by vertex subsets, Linear Algebra Appl. 438, pp. 3278-3290, (2013).

[3] H. Liu, M. Lu, A conjecture on the diameter and signless Laplacian index of graphs, Linear Algebra Appl. 450, pp. 158-174, (2014).

[4] D. Cvetković, P. Rowlinson, S.K. Simić, Eigenvalue bounds for the signless Laplacian, Publications de L'Institute Mathématique, Nouvelle série, tome 81 (95), pp. 11-27, (2007).

[5] P. Hansen, C. Lucas, Bounds and conjectures for the signless Laplacian index of graphs, Linear Algebra Appl. 432, pp. 3319-3336, (2010).

[6] Miao-Lin Ye, Yi-Zheng Fan, Hai Feng Wang, Maximizing signless Laplacian or adjacency spectral radius of graphs subject to fixed connectivity, Linear Algebra Appl. 433, pp. 1180-1186, (2010).

[7] G. Yu, On the maximal signless Laplacian spectral radius of graphs with given matching number, Proc. Japan Acad. Ser. A 84, pp. 163166, (2008). 


\section{Nair Abreu}

Production Engineering Program, PEP/COPPE

Universidade Federal do Rio de Janeiro

Rio de Janeiro,

Brazil

e-mail : nairabreunovoa@gmail.com

\section{Eber Lenes}

Departamento de Investigaciones

Universidad del Sinú. Elías Bechara Zainúm

Cartagena,

Colombia

e-mail: elenes@ucn.cl

and

\section{Oscar Rojo}

Department of Mathematics

Universidad Católica del Norte

Antofagasta,

Chile

e-mail: orojo@ucn.cl 